\title{
The roles of interoceptive sensitivity and metacognitive interoception in panic
}

\author{
Adrián Yoris ${ }^{1,3,4}$, Sol Esteves ${ }^{1}$, Blas Couto ${ }^{1,2,4}$, Margherita Melloni ${ }^{1,2,4}$, Rafael Kichic ${ }^{1,3}$, Marcelo Cetkovich ${ }^{1,3}$, \\ Roberto Favaloro', Jason Moser ${ }^{5}$, Facundo Manes ${ }^{1,2,4,7}$, Agustin Ibanez ${ }^{1,2,4,6,7}$ and Lucas Sedeño $0^{1,2,4^{*}}$
}

\begin{abstract}
Background: Interoception refers to the ability to sense body signals. Two interoceptive dimensions have been recently proposed: (a) interoceptive sensitivity (IS) -objective accuracy in detecting internal bodily sensations (e.g., heartbeat, breathing)-; and (b) metacognitive interoception (MI) -explicit beliefs and worries about one's own interoceptive sensitivity and internal sensations. Current models of panic assume a possible influence of interoception on the development of panic attacks. Hypervigilance to body symptoms is one of the most characteristic manifestations of panic disorders. Some explanations propose that patients have abnormal IS, whereas other accounts suggest that misinterpretations or catastrophic beliefs play a pivotal role in the development of their psychopathology. Our goal was to evaluate these theoretical proposals by examining whether patients differed from controls in IS, MI, or both. Twenty-one anxiety disorders patients with panic attacks and 13 healthy controls completed a behavioral measure of IS motor heartbeat detection (HBD) and two questionnaires measuring MI.
\end{abstract}

Findings: Patients did not differ from controls in IS. However, significant differences were found in MI measures. Patients presented increased worries in their beliefs about somatic sensations compared to controls. These results reflect a discrepancy between direct body sensing (IS) and reflexive thoughts about body states (MI).

Conclusion: Our findings support the idea that hypervigilance to body symptoms is not necessarily a bottom-up dispositional tendency (where patients are hypersensitive about bodily signals), but rather a metacognitive process related to threatening beliefs about body/somatic sensations.

Keywords: Anxiety disorder, Panic attacks, Interoception sensitivity, Metacognitive interoception, Heartbeat detection

\section{Background}

Interoception (the ability to perceive bodily sensations) [1] has been proposed as a risk factor for panic attacks $[1,2]$. Two of its multiple dimensions [3] are related to panic [2]: i) interoceptive sensitivity (IS) -the objective detection of visceral sensations, assessed via tasks such as heartbeat detection (HBD)-, and ii) metacognitive interoception (MI), defined here as participants' reflexive beliefs and thoughts about one's own body sensations.

\footnotetext{
* Correspondence: lucas.sedeno@gmail.com

'Laboratory of Experimental Psychology and Neuroscience (LPEN), INECO (Institute of Cognitive Neurology) and Institute of Neuroscience, Favaloro, Favaloro University, Pacheco de Melo 1860, Buenos Aires C1078AAI,

Argentina

${ }^{2}$ UDP-INECO Foundation Core on Neuroscience (UIFCoN), Diego Portales

University, Santiago, Chile

Full list of author information is available at the end of the article
}

While MI has been restricted to explicit knowledge about accuracy during interoceptive tasks [3], we characterize it as beliefs about bodily sensations at large [4].

IS studies in panic disorders are inconclusive [5], with patients performing either better than [2] or similar to [6] controls (Additional file 1: 1.1). These studies have employed two types of HBD tasks: i) mental tracking paradigms, currently questioned because its working memory demands might affect cardiac perception [7]; and ii) discrimination tasks, where interference generated by simultaneous attention to cardiac sensation and external stimuli may constitute a confound [7]. The possibility that these lurking variables may be the source of discrepant results calls for more robust methods in IS research. 
MI is consistent with cognitive models of panic which emphasize the misinterpretation of somatic sensations as a fundamental aspect of its psychopathogenesis [8], with patients reporting more worries about body signals than controls $[6,9,10]$.

IS and MI constitute different interoceptive processes [3] which are not necessarily associated $[9,10]$. Here, we assessed IS through a novel resting HBD paradigm that addresses certain limitations of other resting cardiac IS tasks [11-13] (see its advantages in Additional file 1: 2.2). MI was examined with self-report measures about body sensation beliefs.

Our overarching hypothesis was that patients and controls would differ in IS and MI. Specifically, we predicted that patients, relative to controls, would perform better in IS and obtain higher scores in MI associated with catastrophic beliefs about body signals.

\section{Methods}

\section{Subjects}

The sample compressed twenty-one anxiety disorder patients [14] who experienced at least one panic attack (PA) $[2,10]$ in the month before testing, and 13 healthy controls. Both groups were matched for age, gender, and education (Table 1). The PA group encompassed different DSM-IV anxiety diagnoses, including panic disorder. We selected this broad-range of diagnoses to assess the underlying mechanisms of panic attacks in anxiety disorders patients. In addition, it has been shown that panic attack episodes are similar to panic attacks in panic disorders [15].
Patients' diagnoses were established with the SCID-I [16] by an anxiety disorder expert, and the presence of panic attacks was established according to Barlow's criteria [17]. Controls had never experienced panic attacks and had no history of drug abuse or neuropsychiatric disease. Body mass index was controlled given its influence on IS [18]. Participants provided informed consent in accordance with the Declaration of Helsinki. The study was approved by INECO institutional ethics committee.

\section{Mood and anxiety measurements}

Mood and depression levels were assessed via the Beck Depression Inventory Second Edition (BDI-II), while state and trait anxiety levels were examined through the State Trait Anxiety Inventory (STAI) (Table 2).

\section{IS: Heartbeat Detection Task (HBD)}

The HBD task is a motor tracking test that assesses IS at rest [11-13]. Participants had to tap a key on a keyboard in rhythm with their heartbeat in different conditions (see Figure 1 and Additional file 1: 2.1 for a detailed explanation).

\section{MI: self-report questionnaires}

Beliefs about body signals were assessed with the Body Sensations Questionnaire (BSQ) and the Physical Concern Index (PCI) of the Agoraphobic Cognitions Questionnaire (ACQ) [19]. Created to target the fear of fear construct, these instruments were here used as an index of catastrophic thoughts about interoceptive sensations [6]. The BSQ measures fear of bodily sensations associated with high arousal and panic. The PCI assesses reflexive

Table 1 Demographic, neuropsychological and clinical results

\begin{tabular}{|c|c|c|c|c|}
\hline & $\mathbf{F}$ & $\mathbf{P}$ & Patients & Control sample \\
\hline Gender & $0.03(x 2)$ & 0.85 & Male $=12 ;$ Female $=9$ & Male $=7 ;$ Female $=6$ \\
\hline Age (years) & 0.00 & 0.97 & $M=32.33 ; S D=10.23$ & $M=32.46 ; S D=10.01$ \\
\hline Formal education (years) & 1.20 & 0.28 & $M=15.24 ; S D=2.02$ & $M=16 ; S D=1.87$ \\
\hline Body mass index & 2.29 & 0.14 & $M=23.56 ; S D=3.28$ & $M=21.80 ; S D=3.12$ \\
\hline Panic Disorder as primary diagnostic & - & - & 13 subjects & - \\
\hline Others Anxiety diagnostics. & - & - & SP (6), SeP (1) and GAD (1) & - \\
\hline Mixed diagnostic & - & - & 1 subject (PA and PTSD). & - \\
\hline Total of Panic Attacks (last 12 months) & - & - & $M=6.0 ; S D=7.90$ & - \\
\hline Total medication sample (\%) & - & - & $47 \%$ & - \\
\hline$B D /-I I$ & 11.29 & $<0.01^{*}$ & $M=15.80 ; S D=11.53$ & $M=4.23 ; S D=5.54$ \\
\hline STAI Trait & 15.78 & $<0.01^{*}$ & $M=47.19 ; S D=12.24$ & $M=32.38 ; S D=6.92$ \\
\hline STAI State & 2.36 & 0.13 & $M=34.14 ; S D=8.93$ & $M=29.92 ; S D=4.19$ \\
\hline$B S Q$ & 42.74 & $<0.01^{*}$ & $M=47.26 ; S D=10.72$ & $M=23.38 ; S D=9.22$ \\
\hline $\mathrm{PCl}$ & 23.79 & $<0.01^{*}$ & $M=2.07 ; S D=0.12$ & $M=1.15 ; S D=0.14$ \\
\hline
\end{tabular}

*indicates significant differences between patients and controls. $\mathrm{M}=$ mean; $\mathrm{SD}=$ standard deviation.

$\mathrm{SP}=$ social phobia; $\mathrm{SeP}=$ specific phobia; $\mathrm{GAD}=$ general anxiety disorders; $\mathrm{PA}=$ panic disorder; $\mathrm{PTSD}=$ post-traumatic stress disorder.

\# Medication details are listed in Additional file 1: 3.7. 
Table 2 Detailed description of self-report questionnaires used

The Beck Depression Inventory-II

The State-Trait Inventory

Body Sensations Questionnaire

Physical Concern Index
(BDI-II) is a 21-item depression scale that assesses emotional, behavioral, and somatic symptoms. Items on the $\mathrm{BDI}-\mathrm{II}$ are rated on a four-category Likert scale that goes from 0 to 3, with a maximum total score of 63. Higher scores indicate more severe depressive symptoms.

(STAI) is a 40 item scale, which assesses both state and trait anxiety and represents a well-validated and reliable self-report measure of dispositional and state anxiety. The scales for trait and state anxiety are made up of 20 items. Participants are asked to indicate to what degree the items describe their dispositional and situational feelings on a four-point Likert-type scale.

(BSQ) is a 17-items scale concerning the degree to which patients fear somatic symptoms commonly associated to panic (i.e. dizziness, heart palpitation, chest pressure). Items are related on five point scales regarding from not frightened or worried by this sensation to extremely frightened by this sensation.

Is a subscale of the Agoraphobic Cognitions Questionnaire (ACQ). It describes thoughts and believes about fear to physical symptoms of anxiety and panic attack. thoughts about physical concerns and their negative consequences (Table 2).

\section{Data analysis}

ANOVA tests were used for demographic and clinical questionnaires. Categorical variables (e.g., gender) were analyzed with the Pearson chi-square $\left(\chi^{2}\right)$ test. Mixed repeated measured ANOVAs were performed for HBD, with a within-subject factor (the seven conditions) and a between-subject factor (the two groups; see Additional file 1: 3.1). Considering the possible influence of depression and anxiety on interoception $[5,20]$ - and the significant differences between groups (Table 1)-, we performed an ANCOVA using BDI and STAI (trait and state) scores as covariates. This analysis was applied only to interoceptive conditions from the HBD task and to self-questionnaires of MI. Effect sizes were reported with partial eta $\left(\eta_{\mathrm{p}}^{2}\right)$.

\section{Results}

Demographic results

No group differences were found in gender $[\times 2(1,34)=$ $0.03, p=0.85]$, age $\left.[F(1,32)<0.01), p=0.97, \eta_{p}^{2}=0.04\right]$, formal education $\left[F(1,32)=1.20, p=0.28, \eta_{p}^{2}=0.03\right]$ or body mass index $\left[F(1,30)=2.29, p=0.14, \eta_{p}^{2}=0.07\right]$.

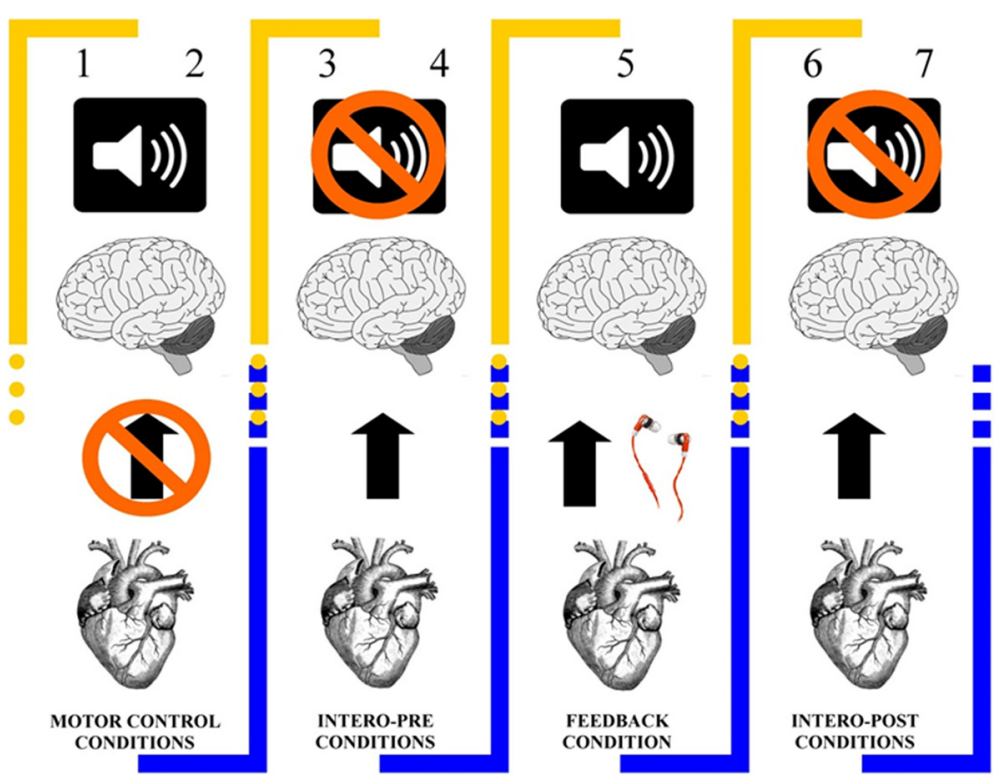

Figure 1 Experimental design of heartbeat detection task (HBD). The HBD task, a motor tracking test, is an experimental procedure in which participants tap a keyboard along with their heartbeats in different conditions (each lasting 2 minutes). First, as motor-control conditions, participants followed an audio-recording of a synchronic heartbeat (1) and then a non-synchronic heartbeat (2). Next, they followed their heartbeats without external feedback (intero-pre conditions) in two intervals ( 3 \& 4). Then, in a feedback control condition, they did the same while receiving simultaneous auditory feedback of their own heart provided through online EKG register (feedback condition), (5). Finally, they followed their own heartbeats without feedback (intero-post conditions) two times (6 \& 7). These conditions offer a measure of audio-motoric performance (first and second conditions), and a cardiac interoceptive measure prior to (intero-pre condition) and after (intero-post condition) the feedback condition. During this task we also measured heart rate (HR) and heart rate variability (HRV) to control their possible influence on IS (details in Additional file 1: 3.5). 


\section{Clinical results}

Group differences for BDI-II $[F(1,31)=11.29, p<0.01$, $\left.\eta_{p}^{2}=0.26\right]$ revealed higher scores of depressive symptoms in PAs than in controls. Both groups showed similar state anxiety levels $\left[F(1,31)=2.36, p=0.14, \eta_{p}^{2}=0.07\right]$. However, patients exhibited significant higher trait anxiety levels $\left[F(1,32)=15.76, p<0.01, \eta_{p}^{2}=0.33\right]$.

\section{Interoceptive sensitivity (IS)}

There was no effect of group $[F(1,26)=1.76, p=0.19$, $\eta p 2=0.06]$ and no interaction between condition and group $[F(6,156)=0.82, p=0.55, \eta p 2=0.03]$. Only an expected [21] and irrelevant effect of condition was observed (Figure 2A and Additional file 1: 3.1). Furthermore, ANCOVA results revealed no differences between interoceptive conditions across groups (Additional file 1: 3.1). No significant differences were found in terms of heart rate (HR) and HRV heart rate variability (HRV) (Additional file 1: 3.5).

\section{Metacognitive interoception (MI)}

Relative to controls, the PA group exhibited higher fear to physical symptoms [BSQ: $F(1,30)=42.74, p<0.01$,

\section{A $\quad$ HBD}

Participants had to tap a keyboard along with their heartbeat in different conditions

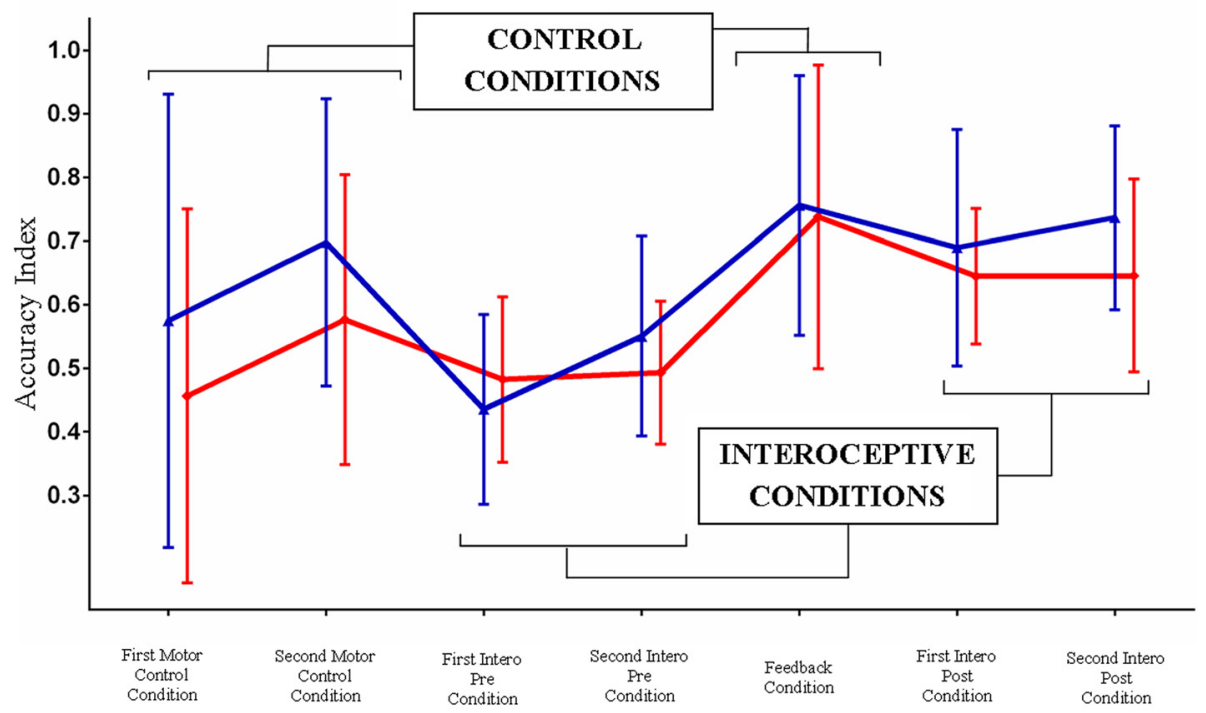

PATIENTS CONTROLS

B

BSQ

Fear to somatic symptoms asociated to panic

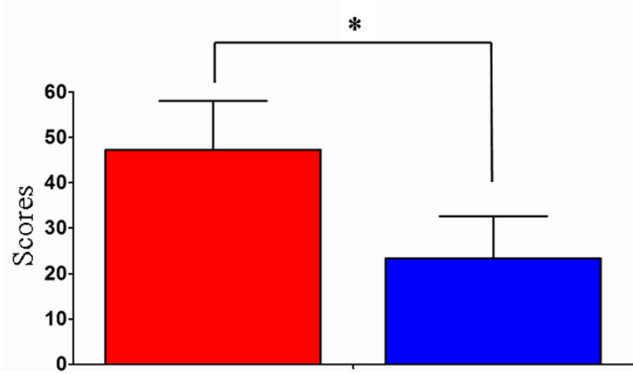

PCI

Physical concerns

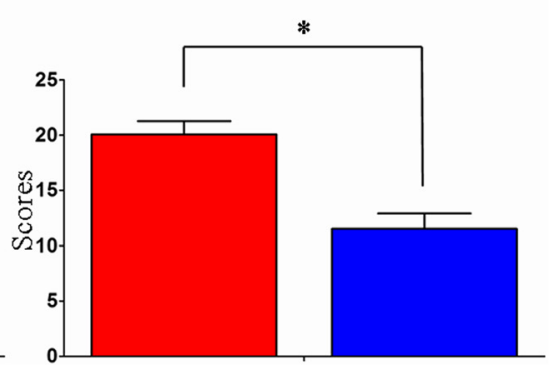

Figure 2 Interoceptive sensitivity (IS): (A) Heartbeat Detection Task (HBD). The Accuracy Index can vary between 0 and 1, with higher scores indicating better accuracy. No differences were found between groups in any condition. Metacognitive interoception (MI): (B) The BSQ indexes the level of worry about body sensations and the PCl assesses cognitions about threatening impact of anxiety bodily symptoms. Both questionnaires yielded significant differences between groups. Vertical bars indicate standard deviations and asterisks signal significant differences. 
$\eta_{p}^{2}=0.58$ ] and higher body anxiety sensations [PCI: $F(1$, $\left.30)=23.79, p<0.01, \eta_{p}^{2}=0.44\right]$ (Figure 2B).

\section{Discussion}

We found no differences in IS between patients and controls. Also, we found evidence for altered MI in patients, who exhibited more worries and catastrophic thoughts about somatic anxiety symptoms.

Previous results of IS in panic populations are inconclusive. They suggest that (i) increased IS is not restricted to panic disorders; (ii) only a small group of patients can be categorized as 'good heart rate perceivers', and (iii) results seem to depend on the paradigm used $[5,22]$. We selected a novel motor resting HBD task that addresses certain methodological limitations of previous reports, such as working memory load and external stimulus interference during interception (Additional file 1: 2.2). Our findings are consistent with the negative results regarding IS [6]. The mental tracking paradigm is the only procedure that has yielded significant differences [2,5], albeit inconsistently. Moreover, accurate heartbeat perception, as measured with this paradigm, is uncommon in both controls and panic patients [6]. Even with the advantages of our new method, no differences were found regarding IS. In addition, the inclusion of BDI and STAI scores as co-factors suggests that these negative results are not affected by such measures (Additional file 1: 3.1 and 3.6).

Together with previous research, our results suggest that this bottom-up process could be a vulnerability factor, but not a pivotal one in the pathogenesis of panic [5].

Regarding MI, our results showed that patients have more worries about body sensations than controls. This is consistent with previous research $[6,9,10]$ and with cognitive models of panic suggesting that the misinterpretation of body signals is a risk factor for panic attacks [8]. In addition, recent prediction coding models of anxiety [23] propose that 'interoceptive prediction schemas' (beliefs and predictions about bodily sensations) produce inaccurate predictions about body signals. Moreover, panic treatments based on the modification of biased threatening beliefs about body symptoms are the most effective ones [24]. Thus, the modification of beliefs about the threatening value of bodily sensations might be a fundamental mechanism underlying effectiveness of cognitive interventions.

In conclusion, the present findings suggest differential contributions of IS and MI to panic attacks. This distinction aligns well with reports showing that both dimensions are not associated [3]. The absence of such correlations in our data corroborates such results (Additional file 1: 3.4).

Two limitations in the present study are its small sample size and the diagnostic variability among patients. Nevertheless, we have reported for the first time the comparison of IS and MI in PA using a more robust HBD paradigm than previous ones. In addition, other studies have found no differences in mixed diagnostic groups [2,9]. Moreover, our results remained the same when considering only patients with panic disorder (see reanalysis in Additional file 1: 3.2). The dissociation between IS and MI suggests that further studies should include a multidimensional interoceptive assessment. Another limitation was that IS was measured at rest. Previous studies have shown increased IS with elevated arousal $[9,22]$. However, our goal was to determine whether classical findings during such a state would be replicated given the demands of our design. Finally, the high proportion of patients under medication could be considered a limitation. Nonetheless, a single-case analysis revealed no effect of medication on IS (Additional file $1: 3.7)$.

\section{Conclusion}

Significant differences were observed only in the beliefs that patients have about somatic sensations but not in their sensitivity to detect them. Considering these results, previous studies, and anxiety models, it seems that IS might be a vulnerability factor for panic attacks. Still, the fundamental mechanism in the pathogenesis of panic attacks might be a tendency to experience somatic/body signals as threatening sensations.

\section{Additional file}

Additional file 1: Additional Methods and Results. In this additional file we provide the following information about: 1) previous studies regarding interoception and panic; 2) a further clinical description of the patients' sample; 3) a detailed description of the Motor Heartbeat Detection Task (HBD) and 4) additional results and conclusions.

\section{Abbreviations}

(IS): Interoceptive sensitivity; (MI): Metacognitive interoception; (HBD): Heartbeat detection task; (PA): Anxiety patients that experienced at least one panic attack episode; (SCID-I): Structured Clinical Interview for DSM-IV; (BDI): Beck's Depression Inventory; (STAI): State Trait Anxiety Inventory; (BSQ): The Body Symptoms Questionnaire; (ACQ): Agoraphobic Cognitions Questionnaire; (PCI): Physical Concern Index.

\section{Competing interests}

The authors declare that they have no competing interests.

\section{Authors' contributions}

AY and LS collected the data, statistically analyzed the data, and wrote the first draft of the manuscript. MM collected the data. SE statistically analyzed the data and wrote the first draft of the manuscript. BC was involved in the study conception and design, writing the protocol, and contributed to the manuscript draft. RK contributed in collecting the data and revising the final version of the manuscript. RF, JM, MC and FM contributed to revising the final version of the manuscript. Al was involved in the study conception and design and contributed to writing the final version of the manuscript. All authors read and approved the final manuscript.

\section{Acknowledgements}

This research was partially supported by CONICET, INECO Foundation, CONICYT/FONDECYT Regular (1130920), FONCYT- PICT 2012-0412, FONCyT- 
PICT 2012-1309. Any opinions, findings, and conclusions or recommendations expressed in this material are those of the authors and do not necessarily reflect the views of those grants.

\section{Author details}

${ }^{1}$ Laboratory of Experimental Psychology and Neuroscience (LPEN), INECO (Institute of Cognitive Neurology) and Institute of Neuroscience, Favaloro, Favaloro University, Pacheco de Melo 1860, Buenos Aires C1078AAl, Argentina. ${ }^{2}$ UDP-INECO Foundation Core on Neuroscience (UIFCoN), Diego Portales University, Santiago, Chile. ${ }^{3}$ Anxiety and Trauma Clinic, INECO (Institute of Cognitive Neurology), C1078AAI Buenos Aires, Argentina. ${ }^{4}$ National Scientific and Technical Research Council (CONICET), Buenos Aires, Argentina. ${ }^{5}$ Department of Psychology, Michigan State University, East Lansing, MI, USA. ' Universidad Autónoma del Caribe, Barranquilla, Colombia. ${ }^{7}$ Australian Research Council (ACR) Centre of Excellence in Cognition and its Disorders, Macquarie University, NSW 2109 Sydney, Australia.

Received: 3 October 2014 Accepted: 4 March 2015

Published online: 08 April 2015

\section{References}

1. Craig AD. How do you feel? Interoception: the sense of the physiological condition of the body. Nat Rev Neurosci. 2002;3:655-66.

2. Ehlers A, Breuer P. Increased cardiac awareness in panic disorder. J Abnorm Psychol. 1992;101:371-82.

3. Garfinkel SN, Critchley HD. Interoception, emotion and brain: new insights link internal physiology to social behaviour. Commentary on: "Anterior insular cortex mediates bodily sensibility and social anxiety" by Terasawa et al. (2012). Soc Cogn Affect Neurosci. 2013;8:231-4.

4. Clark DM, Salkovskis PM, Ost LG, Breitholtz E, Koehler KA, Westling BE, et al. Misinterpretation of body sensations in panic disorder. J Consult Clin Psychol. 1997;65:203-13.

5. Domschke K, Stevens S, Pfleiderer B, Gerlach AL. Interoceptive sensitivity in anxiety and anxiety disorders: an overview and integration of neurobiological findings. Clin Psychol Rev. 2010;30:1-11.

6. Van Der Does WAJ, Van Dyck DR, Spinhoven P. Accurate heartbeat perception in panic disorder: Fact and artefact. J Affect Disord. 1997:43:121-30.

7. Richards JCE, Lorraine V. Cardiac acuity in panic disorder. Cogn Therapy Res. 1996;20:361.

8. Clark DM. A cognitive approach to panic. Behav Res Ther. 1986;24:461-70.

9. Antony M, Brown T, Craske M, Barlow D, Mitchell W, Meadows E. Accuracy of heartbeat perception in panic disorder, social phobia, and nonanxious subjects. J Anxiety Disord. 1995;9:355-71.

10. Zoellner LA, Craske MG. Interoceptive accuracy and panic. Behav Res Ther. 1999;37:1141-58.

11. Sedeno L, Couto B, Melloni M, Canales-Johnson A, Yoris A, Baez S, et al. How do you feel when you can't feel your body? Interoception, functional connectivity and emotional processing in depersonalization-derealization disorder. PLoS One. 2014;9:e98769.

12. Melloni M, Sedeno L, Couto B, Reynoso M, Gelormini C, Favaloro R, et al. Preliminary evidence about the effects of meditation on interoceptive sensitivity and social cognition. Behav Brain Functions BBF. 2013;9:47.

13. Couto B, Salles A, Sedeno L, Peradejordi M, Barttfeld P, Canales-Johnson A, et al. The man who feels two hearts: the different pathways of interoception. Soc Cogn Affect Neurosci. 2014;9:1253-60.

14. American Psychiatric Asociation A. Diagnostic and Statistical Manual of Mental Disorders. New York: Masson; 1994.

15. Ehlers A, Breuer P. How good are patients with panic disorder at perceiving their heartbeats? Biol Psychol. 1996;42:165-82.

16. First MB, Spitzer RL, Gibbon M, Williams JB. Structured Clinical Interview for DSM-IVA Axis I Disorders, Research Version, Patient Edition (SCID-I/P). New York: Biometrics Research, New York State Psychiatric Institute; 2002.

17. Barlow DH, Brown TA, Craske MG. Definitions of panic attacks and panic disorder in the DSM-IV: implications for research. J Abnorm Psychol. 1994;103:553-64

18. Rouse $\mathrm{CH}$, Jones $\mathrm{GE}$, Jones KR. The effect of body composition and gender on cardiac awareness. Psychophysiology. 1988;25:400-7.

19. Chambless DL, Caputo GC, Bright P, Gallagher R. Assessment of fear of fear in agoraphobics: the body sensations questionnaire and the agoraphobic cognitions questionnaire. J Consult Clin Psychol. 1984;52:1090-7.
20. Dunn BD, Dalgleish T, Ogilvie AD, Lawrence AD. Heartbeat perception in depression. Behav Res Ther. 2007;45:1921-30.

21. Van der Does A, Antony M, Ehlers A, Barsky A. Heartbeat perception in panic disorder: a reanalysis. Behav Res Ther. 2000;38:47-62.

22. Paulus MP, Stein MB. Interoception in anxiety and depression. Brain Struct Funct. 2010;214:451-63.

23. Barlow DH. Cognitive-behavioral therapy for panic disorder: current status. J Clin Psychiatry. 1997;58 Suppl 2:32-6. discussion 36-37.

24. Boswell JF, Farchione TJ, Sauer-Zavala S, Murray HW, Fortune MR, Barlow $\mathrm{DH}$. Anxiety sensitivity and interoceptive exposure: a transdiagnostic construct and change strategy. Behav Ther. 2013;44:417-31.

\section{Submit your next manuscript to BioMed Central and take full advantage of:}

- Convenient online submission

- Thorough peer review

- No space constraints or color figure charges

- Immediate publication on acceptance

- Inclusion in PubMed, CAS, Scopus and Google Scholar

- Research which is freely available for redistribution 